\title{
Linear and Volumetric Analysis of Maxillary Sinus Pneumatization in a Sri Lankan Population Using Cone Beam Computer Tomography
}

\author{
Pilana Vithanage Kalani Shihanika Hettiarachchi $\mathbb{D}^{1}$, \\ Pulihinga Mudiyanselage Prabhath Chaminda Gunathilake, ${ }^{2}$ Rasika Manori Jayasinghe $\mathbb{D}^{3}{ }^{3}$ \\ Manil Christopher Fonseka, ${ }^{4}$ Ranasinghe Mudiyanselage Wikum Roshan Bandara $\mathbb{D}^{1}{ }^{1}$ \\ Chinthani Deepthi Nanayakkara, ${ }^{5}$ and Ruwan Duminda Jayasinghe $\mathbb{D}^{1}$ \\ ${ }^{1}$ Department of Oral Medicine and Periodontology, Faculty of Dental Sciences, University of Peradeniya, Sri Lanka \\ ${ }^{2}$ Department of Statistics and Computer Science Faculty of Science, University of Peradeniya, Sri Lanka \\ ${ }^{3}$ Department of Prosthodontics, Faculty of Dental Sciences, University of Peradeniya, Peradeniya, Sri Lanka \\ ${ }^{4}$ Department of Restorative Dentistry, Faculty of Dental Sciences, University of Peradeniya, Peradeniya, Sri Lanka \\ ${ }^{5}$ Department of Basic Sciences, Faculty of Dental Sciences, University of Peradeniya, Sri Lanka
}

Correspondence should be addressed to Pilana Vithanage Kalani Shihanika Hettiarachchi; kalaniz2004@yahoo.com

Received 16 October 2020; Revised 25 January 2021; Accepted 30 March 2021; Published 8 April 2021

Academic Editor: Xiao Lei Zhang

Copyright (c) 2021 Pilana Vithanage Kalani Shihanika Hettiarachchi et al. This is an open access article distributed under the Creative Commons Attribution License, which permits unrestricted use, distribution, and reproduction in any medium, provided the original work is properly cited.

\begin{abstract}
Objective. The objectives of this study were to evaluate the linear and volumetric measurements of the maxillary sinus in relation to sex and side on cone beam computer tomographic (CBCT) images in a Sri Lankan population. Methods. A total of 20 sets of CBCT images selected from the database at the Division of Oral Medicine and Radiology, Faculty of Dental Sciences, University of Peradeniya, Sri Lanka, were evaluated. Linear measurements were obtained in a craniocaudal (height), anteroposterior (length), and mediolateral (width) dimensions. Volume was computed by using the same data using a computerized 3D modeling software developed for 3D measurements and calculations. Results. The maximum mean craniocaudal dimension was at the level of the 1st and the 2nd molar tooth bilaterally. The largest average craniocaudal, mediolateral, and anteroposterior extensions of the maxillary sinus using CBCT were $31.71 \pm 5.44 \mathrm{~mm}, 21.28 \pm 5.09 \mathrm{~mm}$, and $32.92 \pm 4.31 \mathrm{~mm}$, respectively. The differences between the sides and sex showed no statistical significance $(P>0.05)$, except for the maximum average value in craniocaudal dimension which showed a statistically significant difference in relation to gender $(P=0.02)$. Conclusion. There is no significant difference in the largest average craniocaudal, mediolateral, and anteroposterior extensions of the maxillary sinus when gender and side were compared. However, the maximum average value in craniocaudal dimension had a statistically significant difference in relation to gender. This study provides valuable knowledge of the anatomical dimensions of the maxillary sinus which may help clinicians in treatment planning.
\end{abstract}

\section{Introduction}

Dental implant placement has revolutionized the dentistry in the recent past has resulted in transforming our skills as oral health care practitioners. The censorious structure within the posterior mandible is the inferior alveolar nerve which must be avoided when an implant is placed. Similarly, the sinus is the important structure situated within the maxilla, which can limit the utilization of implants causing complications.

The largest of the paranasal sinuses is the maxillary sinuses, and it was first described by Leonardo da Vinci in 1489 and later documented as "antrum of Highmore" following its description by an English anatomist, Nathaniel Highmore in $1651[1,2]$. 
The volume of this pyramidal shape structure usually measures $15 \mathrm{cc}$; however, the value may vary with age, gender, and ethnicity $[3,4]$. In addition, dimensions of the maxillary sinus tend to change due to loss or absence of teeth as well. Identification of proper linear and volumetric measurements of the maxillary sinus in a given population is clinically important as it can guide the clinician in proper decision-making during various treatment procedures. Cone-beam computed tomography (CBCT), since the introduction into routine clinical practice, is frequently used to evaluate sinus anatomy especially prior to dental implant treatment $[5,6]$. It facilitates evaluation of the maxillofacial region with high-geometric-accuracy data, isotropic voxel values, and short scanning times [5, 7]. In addition, it provides increased precision, lower radiation doses, and lower costs than computed tomography (CT) $[7,8]$. Further, CBCT is an efficient alternative to standard multidetector computed tomography (MDCT) to identify sinusitis as the image quality and radiation dose are comparable to MDCT [9].

In spite of being an important anatomical structure, limited availability of literature together with marked population variations [10] warrants the necessity to evaluate parameters in relation to maxillary sinus especially in the local population. Hence, the present study was undertaken to ascertain the linear and volumetric measurements of the maxillary sinus in relation to sex and side on CBCTs in a Sri Lankan population.

\section{Methods}

This study was performed as a retrospective analysis of data obtained from the archives of the Division of Oral Medicine and Radiology of the Dental Teaching Hospital, Peradeniya, Sri Lanka. Deidentified images of twenty maxillary sinuses belonging to 10 patients between the 20-30 years of age were analyzed to determine the dimensions of the maxillary sinuses. Images of patients with incomplete clinical records, distorted images, previous history of maxillary surgery, gross malocclusions, or cranio-facial anomalies and patients with cleft lip and palate, maxillofacial trauma, and maxillary arches with edentulous areas involving more than two posterior teeth and sinuses with mucosal thickening were excluded from the study. Ethical clearance was obtained from the Ethics Review Committee, Faculty of Dental Sciences, University of Peradeniya, and written consent had been obtained from all the participants for using the data for study purposes prior to image acquisition.

All CBCT images were acquired using a Vatech, PaXDuo3D CBCT scanner (Vatech Corporation, South Korea) using a range of $18-200 \mathrm{uSV}, 60$ to $90 \mathrm{kvp}$, and $2-15 \mathrm{~mA}$ allowing any adjustment within each FOV and voxel size under standard settings. Images were stored and converted to a DICOM format using the acquisition software integrated to the above CBCT machine. The linear measurements were obtained using EzDent software measurement tools with precision values of $0.1 \mathrm{~mm}$, while the area and the volume were computed by using the same DICOM images in a computerized 3D modeling software [11].
2.1. Linear Measurement Determination. Parameters described by Hamdy et al. (2014) were mostly used in the study considering the anatomical fact that the maxillary sinus is pyramidal in shape, with an almost square base that is oriented medially [12]. The measurements of the sinus dimensions were conducted using the methods described by Hamdy et al. in 2014 [12] as the guide.

(1) Craniocaudal (CC) measurements are depicted in Figure 1

Bilateral measurements between upper first and second premolars, upper second premolar and upper first molar, upper first and second molars, and upper second and third molars were obtained (CC4-5, CC5-6, CC6-7, and CC7-8, respectively).

(2) Anteroposterior (AP) and mediolateral dimension (ML) (Figure 2)

Measurements were carried out at two levels: along the nasal floor and along the root of zygoma resulting in a total of 4 different measurements (AP Nasal, AP Zygoma, ML Nasal, and ML Zygoma), respectively, on each side.

2.2. Sinus Volume Determination. Volumetric analysis was conducted for all the sinuses utilized to obtain linear measurements. The pipeline process of isolating the $3 \mathrm{D}$ volume of each sinus is described below.

(a) In each set of images, the range of image slices attached with sinus volume was isolated manually by looking at the complete range of images

(b) To separate the sinus region from other parts of each image, an identified threshold was assigned. The separated sinus area was coloured black, while the background was set to white

(c) A 3D wireframe was generated using the threshold applied images as shown in Figure 3. The covering volume was calculated with the information available from the input DICOM files. The pixel to millimeter ratio of image slices was found as 1:0.4726564

\section{Results}

All linear and volumetric measurements were performed by two investigator separately. To assess intraexaminer reliability, 10 maxillary sinuses were randomly selected, and all measurements were repeated. These measurements were made after a reasonable time interval. The two sets of measurements were then compared with paired $t$-tests. Out of twenty CBCT images analyzing maxillary sinuses, the male and female distribution was equal, with a mean age of $24.9 \pm 3.26$ years.

3.1. Linear Measurements of the Maxillary Sinus Height (CC). The maximum CC dimension of the maxillary sinus was observed in between the $1^{\text {st }}$ and the $2^{\text {nd }}$ molar (CC6-7) with a mean measurement of $31.71 \pm 5.44 \mathrm{~mm}$, and $50 \%$ of the 


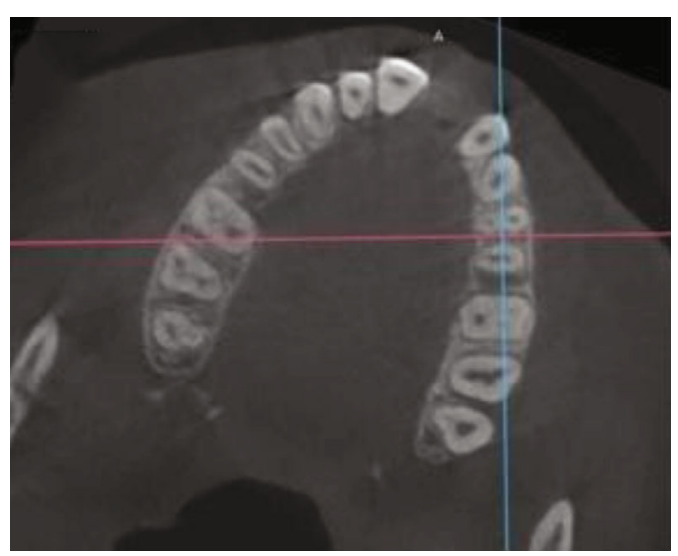

(a)

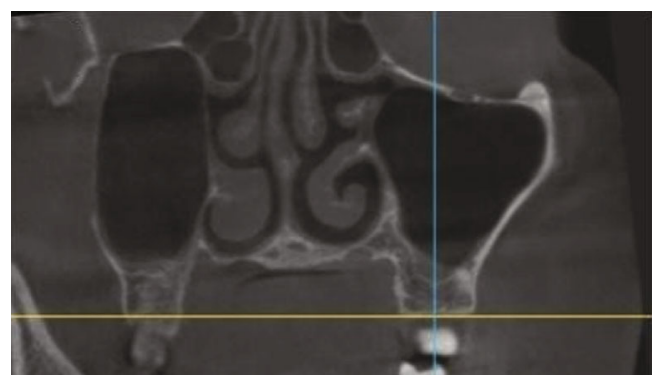

(b)

FIgURE 1: (a) Adjustment of axial cut at area of intended measurement by rotation of the axial image till the orientation axis for the coronal cut (pink line) becomes perpendicular on buccal cortex for each side. (b) Coronal cut revealing the actual craniocaudal measurement conducted along the orientation axis for the sagittal cut (blue line).

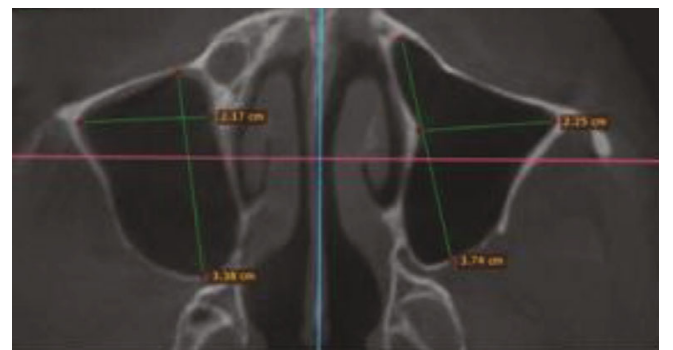

(a)

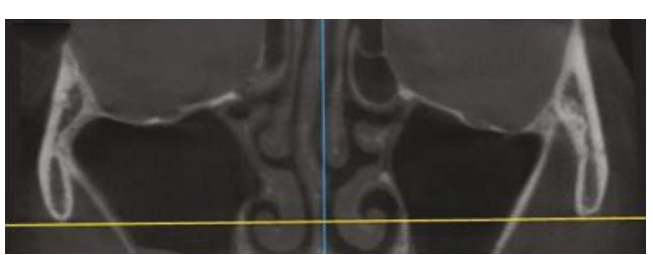

(b)

FIGURE 2: (a) Anteroposterior and mediolateral (1 and 3) measurements conducted along the root of zygoma level (AP ZG, ML ZG) on axial CBCT scan. (b) Coronal CBCT scan show the axial orientation axis (yellow horizontal line) denoting the level of axial scan along the root of zygoma.

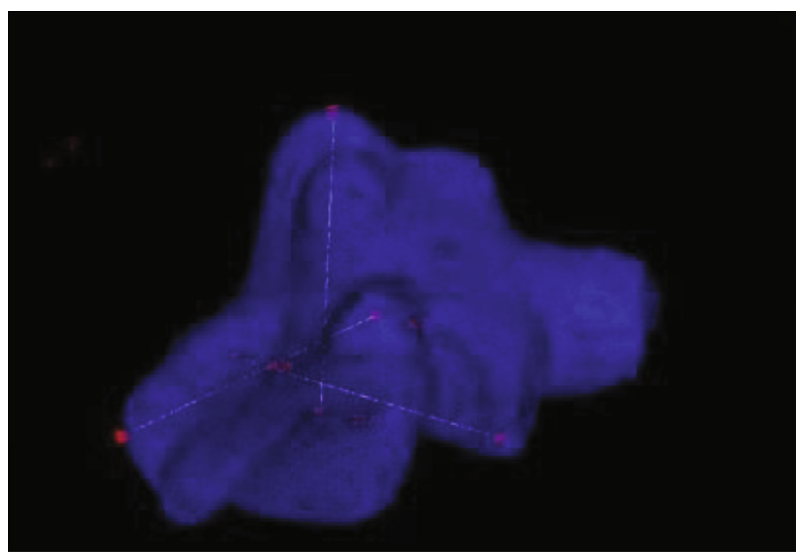

FIGURE 3: Snapshot showing measurements and calculation of the sinus volume, performed by the 3D modelling software [11].

cases had the maximum CC dimensions at the CC 6-7. The measurements at CC 6-7 ranged from $24.6 \mathrm{~mm}$ to $40.9 \mathrm{~mm}$ on the left side with a mean of $33.09 \pm 5.71 \mathrm{~mm}$ and $25.1 \mathrm{~mm}$ to $37.3 \mathrm{~mm}$ on the right side with a mean value of $31.24 \pm 4.09 \mathrm{~mm}$. In almost all cases, the maxillary sinus showed the least CC extension between the $1^{\text {st }}$ and the $2^{\text {nd }}$ premolar (CC 4-5) with a mean of $24.85 \pm 4.72 \mathrm{~mm}$ for the left side and $23.76 \pm 4.89 \mathrm{~mm}$ on the right side (Table 1). However, it was interesting to note that at all levels, the mean craniocaudal dimensions of the females were lesser than the mean values of the males, and the difference between the two genders in maximum mean dimension at the $1^{\text {st }}$ and the $2^{\text {nd }}$ molar (CC6-7) was statistically significant at a $P$ value of $0.02(P<0.05)$, but the minimum mean dimensions at the $1^{\text {st }}$ and the $2^{\text {nd }}$ premolar (CC 4-5) was not statistically significant ( $P$ value of 0.18$)$.

3.2. Linear Measurements of the Maxillary Sinus Length and Width ( $A P$ and $M L$ ). The maximum mean value for the anteroposterior and mediolateral measurements was $32.92 \pm$ $4.31 \mathrm{~mm}$ and $21.28 \pm 5.09 \mathrm{~mm}$, respectively, at the root of the zygoma. Out of all four measurements to assess the length and height of the sinus, it was noted that bilaterally, the maximum mean length was observed at the root of the zygoma antero-posteriorly (right side $32.02 \pm 5.02 \mathrm{~mm}$; left side $33.82 \pm 3.48 \mathrm{~mm} ; P=0.36)$. The maximum mean height in ML direction was again at the root of the zygoma (right side $21.24 \pm 5.42 \mathrm{~mm}$; left side $21.33 \pm 5.02 \mathrm{~mm} ; P=0.96)$. The minimum mean height was observed mediolaterally and bilaterally along the nasal floor. However, none of the 
TABLE 1: Linear measurements of the maxillary sinus, width, length, and height-denoting extension in CC, AP, and ML directions at different levels.

\begin{tabular}{|c|c|c|c|c|c|c|c|c|c|c|c|c|c|c|c|c|}
\hline & \multicolumn{8}{|c|}{ Left side } & \multicolumn{8}{|c|}{ Right side } \\
\hline & \multicolumn{4}{|c|}{ Maxillary sinus width } & \multicolumn{4}{|c|}{$\begin{array}{l}\text { Maxillary sinus height } \\
\text { and length }\end{array}$} & \multicolumn{4}{|c|}{ Maxillary sinus width } & \multicolumn{4}{|c|}{$\begin{array}{l}\text { Maxillary sinus height } \\
\text { and length }\end{array}$} \\
\hline & CC 4-5 & CC 5-6 & CC 6-7 & CC 7-8 & $\begin{array}{l}\text { ML } \\
\text { NS }\end{array}$ & $\begin{array}{c}\mathrm{ML} \\
\mathrm{ZG}\end{array}$ & $\begin{array}{l}\text { AP } \\
\text { NS }\end{array}$ & $\begin{array}{l}\text { AP } \\
\text { ZG }\end{array}$ & CC 4-5 & CC 5-6 & CC 6-7 & CC 7-8 & $\begin{array}{l}\text { ML } \\
\text { NS }\end{array}$ & $\begin{array}{l}\mathrm{ML} \\
\mathrm{ZG}\end{array}$ & $\begin{array}{l}\text { AP } \\
\text { NS }\end{array}$ & $\begin{array}{l}\text { AP } \\
\text { ZG }\end{array}$ \\
\hline 01 & 31.3 & 36.1 & 34.6 & 36.8 & 22.6 & 24.4 & 36.1 & 37.3 & 32.6 & 37.5 & 37.3 & 36.2 & 21.3 & 23.2 & 31.3 & 34.9 \\
\hline 02 & 21.8 & 36 & 35.5 & 34.3 & 14.3 & 20 & 28.3 & 30 & 26.4 & 31.7 & 33.2 & 37.5 & 13.1 & 19.6 & 20.2 & 24.2 \\
\hline 03 & 25.3 & 37.8 & 40.9 & 35.9 & 18.4 & 20 & 32.6 & 33.9 & 22.2 & 34.5 & 33.5 & 23.2 & 15.7 & 19.4 & 24.9 & 26.6 \\
\hline 04 & 26.2 & 33.4 & 38.7 & 34.2 & 23.6 & 28.5 & 35.4 & 38.5 & 21.8 & 33.1 & 34 & 34 & 20.5 & 26.3 & 35.2 & 37.8 \\
\hline 05 & 23.7 & 26.8 & 24.6 & 30.8 & 14.5 & 13 & 11.9 & 29.2 & 18.4 & 28 & 31.1 & 27.2 & 11.8 & 14.1 & 12.1 & 30.6 \\
\hline 06 & 29.6 & 28.2 & 28.4 & 30.8 & 16.6 & 23.2 & 35.4 & 36.4 & 27.9 & 26.1 & 29.6 & 33.1 & 16.1 & 23.9 & 32.1 & 38.7 \\
\hline 07 & 30.4 & 35.7 & 40.3 & 34.7 & 20.5 & 22.2 & 36.2 & 36.2 & 28.8 & 34.7 & 35.2 & 30.9 & 17.4 & 22.4 & 37 & 36.6 \\
\hline 08 & 19.7 & 26.5 & 28.9 & 28.7 & 18.6 & 17.4 & 23.4 & 30.2 & 18.6 & 22.1 & 26.5 & 26.7 & 17.5 & 15.7 & 21.5 & 26.8 \\
\hline 09 & 16.8 & 26.1 & 28.2 & 25.9 & 14 & 16.3 & 28.5 & 30.7 & 19.1 & 27 & 25.1 & 26.6 & 10.5 & 16 & 22.2 & 31.7 \\
\hline 10 & 23.7 & 22.7 & 30.8 & 28.4 & 10.5 & 28.3 & 25.1 & 35.8 & 21.8 & 24.2 & 26.9 & 26.3 & 20.4 & 31.8 & 24.7 & 32.3 \\
\hline Mean & 24.85 & 30.93 & 33.09 & 32.05 & 17.36 & 21.33 & 29.29 & 33.82 & 23.76 & 29.89 & 31.24 & 30.17 & 16.43 & 21.24 & 26.12 & 32.02 \\
\hline $\mathrm{SD}$ & 4.72 & 5.41 & 5.71 & 3.64 & 4.15 & 5.02 & 7.72 & 3.48 & 4.89 & 5.11 & 4.09 & 4.84 & 3.75 & 5.42 & 7.71 & 5.02 \\
\hline $95 \%$ CI & 3.01 & 3.45 & 3.64 & 2.32 & 2.97 & 3.59 & 5.52 & 2.49 & 3.49 & 3.66 & 2.93 & 3.47 & 2.68 & 3.88 & 5.52 & 3.59 \\
\hline
\end{tabular}

differences observed on the right and left side was statistically significant at $P<0.05$ (Table 1 ). In contrast to the CC measurements, all the mean of the lengths and heights were higher in sinuses belonging to females compared with the males.

The mean maximum and minimum measurements were comparable within both genders with the general findings and were on par with the craniocaudal measurements. Further, neither the maximum nor the minimum measurement differences with regard to gender showed a statistically significant difference at $P$ value of 0.05 (Table 2).

3.3. Volumetric Analysis (Figure 4). With regard to the measurement of the volume, the average volume of the right side was slightly more than the average value of the left side (right side-14.77 $\pm 4.55 \mathrm{~cm}^{3}$; left side $-14.74 \pm 4.15 \mathrm{~cm}^{3}$ ), and the males had higher values compared to the females. However, none of these differences was statistically significant $(P=0.89)$.

\section{Discussion}

There is great variation of the pneumatization of the maxillary sinus within the maxilla. This complexity is brought about as a result of differences in the extension of the maxillary sinus. Therefore, the relationship of the sinus floor with the teeth and the alveolar bone in dentate and edentulous ridge, respectively, is very crucial in the decision-making for variety of dental procedures. Such procedures may range from a simple extraction of a maxillary posterior teeth to surgical interventions including oral surgical procedures such as performing a sinus floor augmentation and planning of a dental implant in the posterior maxilla. The outcome of the dental procedure may largely depend on the assessment of volume of the maxillary sinus, and when one fails, it may give rise to a number of complications such as perforation of the sinus floor or may end up with chronic sinusitis. Literature reports seem to be controversial with regard to the changes in the size of the maxillary sinus following dental extractions where some authors witnessed an increase in the size of the maxillary sinus following tooth extraction [13]. Others oppose this fact claiming that there is no such correlation between the dental status and the volume of the maxillary sinus [14]. However, Sharah et al. in 2008 [13] identified that postextraction pneumatization occurred within the socket healing period of 4 to 6 months, and this process may reduce to a minimum or stop completely following the development of mature bone in the extraction socket.

According to the $3 \mathrm{D}$ volumetric segmentation, demonstrated by Hamdy et al., in 2012 [15], the linear measurements performed in this study represent the width, length, and the height of the sinus (from the measurements done on mediolateral anteroposterior and craniocaudal direction, respectively). A retrospective study by Hamdy and AbdelWahed [12], analyzing $30 \mathrm{CBCT}$ scans reported that the maximum craniocaudal, mediolateral, and anteroposterior dimensions were observed around $2^{\text {nd }}$ molar (93\%) and at the level of the root of zygomatic complex in $93 \%$ and $90 \%$ sinuses, respectively. These findings were comparable with results of the present study. In this study, it was found that the largest average craniocaudal, mediolateral, and anteroposterior extensions of the maxillary sinus using CBCT were $31.71 \pm 5.44 \mathrm{~mm}, 21.28 \pm 5.09 \mathrm{~mm}$, and $32.92 \pm 4.31 \mathrm{~mm}$, respectively. Our results demonstrated that the height of this pyramid (mediolateral) is the smallest dimension, while the anteroposterior and craniocaudal dimensions of its base are nearly equal. These findings are comparable with the average dimensions reported by the sinus studies by Tiwana et al. [16] and Hamdy and Abdel-Wahed et al. [12]. Further, according to Tiwana et al., the average dimensions of the 
TABLE 2: The maximum and minimum linear measurements of the maxillary sinuses against the gender.

\begin{tabular}{lcccccc}
\hline & \multicolumn{2}{c}{ Maxillary sinus width } & \multicolumn{2}{c}{ Maxillary sinus length } & \multicolumn{2}{c}{ Maxillary sinus height } \\
& $\begin{array}{c}\text { Maximum } \\
\text { mean } \pm \text { SD }(\mathrm{mm})\end{array}$ & $\begin{array}{c}\text { Minimum } \\
\text { mean } \pm \mathrm{SD}(\mathrm{mm})\end{array}$ & $\begin{array}{c}\text { Maximum } \\
\text { mean } \pm \mathrm{SD}(\mathrm{mm})\end{array}$ & $\begin{array}{c}\text { Minimum } \\
\text { mean } \pm \text { SD }(\mathrm{mm})\end{array}$ & $\begin{array}{c}\text { Maximum } \\
\text { mean } \pm \mathrm{SD}(\mathrm{mm})\end{array}$ & $\begin{array}{c}\text { Minimum } \\
\text { mean } \pm \mathrm{SD}(\mathrm{mm})\end{array}$ \\
\hline Male & $34.59 \pm 4.49$ & $25.71 \pm 5.00$ & $32.02 \pm 5.02$ & $26.12 \pm 7.71$ & $21.24 \pm 5.42$ & $16.43 \pm 3.75$ \\
Female & $29.74 \pm 4.24$ & $22.90 \pm 4.17$ & $33.82 \pm 3.48$ & $29.29 \pm 7.72$ & $21.33 \pm 5.02$ & $17.36 \pm 4.15$ \\
$P$ value & 0.02 & 0.18 & 0.99 & 0.37 & 0.97 & 0.6 \\
\hline
\end{tabular}

SD: standard deviation; mm: millimeters.

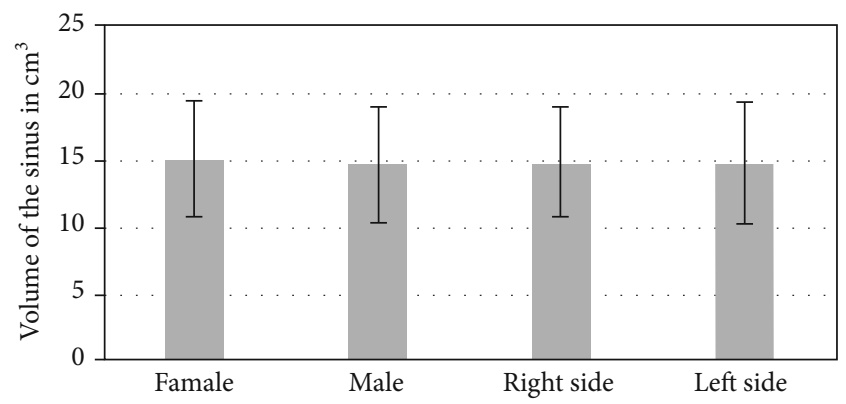

Figure 4: Mean volume of sinuses against the side and gender.

sinus are $33 \mathrm{~mm}$ high, $23 \mathrm{~mm}$ wide, and $34 \mathrm{~mm}$ in anteriorposterior length [16].

Further, the results obtained in the present study demonstrated that only $50 \%$ of the cases had the maximum craniocaudal extension of the maxillary sinus located around the 1 st and 2nd molar ( 10 cases out of $20=50 \%$ ). This result is similar to the studies by Koppe et al. [17] who observed prominences on maxillary sinus floor due to the apices of the first and second maxillary molars in half of its samples. Further, in par with this finding, Ariji et al. [18] reported to observe $60 \%$ of the roots of the maxillary first molar close to the sinus floor. However, this finding contrasts to the ones reported by Nimigean et al. [19], as he reported in majority the lowest point of the maxillary sinus is in relation to the second molar (93.9\%). The variations of the sinus floor's depth will depend on sinus dimensions, their size, and pneumatization [19].

A study conducted by Saccucci et al. [20] to determine the relationship between gender and maxillary sinus volumes included 52 patients ( 26 males and 26 females) with a mean age of 24.3 years revealed that there is no statistically significant difference in the patients' maxillary sinus volumes across genders. The results of our study also confirm this fact as there was no statistically significant difference in the linear and volumetric measurements except in linear measurement (craniocaudal) between the 1 st and 2 nd molar teeth. However, a study by Georgiev et al. [21] has revealed a statistically significant difference in favor of male patients in their analysis of volumetric measurements across genders.

The present study, based on a special software application, facilitated a quick and easy estimate of the volume of the maxillary sinus, which has an irregular shape and thus defies accurate mathematical calculation. The volumetric assessment of the maxillary sinus and its pneumatization (primary or secondary) allows clinicians to accurately determine the exact site of surgical intervention and its anatomical variation. Maxillary sinus floor augmentation procedures with lateral access require precise location of the bone window as well as a sufficiently wide sinus, so that elevation or removal could be adequate, and determination of the volume of bone graft material is required for sinus grafting procedures.

\section{Conclusions}

Within the limitations of the present study, the findings highlighted the anatomic variability of the maxillary sinus in relation to several parameters. In comparison to the figures reported in literature, certain aspects of the morphometrics of maxillary sinus in a Sri Lankan population are different from the standard measurements reported for other population groups. Utilization of a cost-effective software for the volumetric assessment is also encouraged especially in the Sri Lankan context. Further studies with larger numbers of CBCT images covering all age groups and ethnic groups will be needed for more information in the Sri Lankan population.

\section{Data Availability}

Access to research data is restricted as these contain patient records.

\section{Ethical Approval}

Ethical approval has been obtained from the ERC of Faculty of Dental Sciences, University of Peradeniya.

\section{Consent}

Informed consent was obtained from all participants. All authors have consented for publication by signing the certification form; however, the participant images are deidentified prior publication. All procedures followed were in accordance with the ethical standards of the responsible committee on human experimentation (institutional and national) and with the Helsinki Declaration of 1964 and later versions. Informed consent was obtained from all patients for being included in the study. However, identifying information of patients or human subjects is not included in the article. 


\section{Conflicts of Interest}

The authors declare that they have no conflict of interest.

\section{Authors' Contributions}

Hettiarachchi is responsible for the data analysis and manuscript writing; Gunatilake for data collection and manuscript editing; M. Jayasinghe and Fonseka for protocol writing and manuscript editing; Bandara for data collection; and Nanayakkara and R. Jayasinghe for protocol development and manuscript editing.

\section{References}

[1] C. E. Misch, Ed., "Dental Implant Prosthetics (Second Edition): Maxillary Posterior Edentulism: Treatment Options for Fixed Prostheses," in Dental Implant Prosthetics (Second Edition)pp. 2015553-2015572, Mosby.

[2] W. H. Hollinshed, Anatomy for Surgeons: The Head and Neck, Lippincott, Philadelphia, 1982.

[3] S. U. Rani, G. V. Rao, D. R. Kumar, T. Sravya, Y. Sivaranjani, and M. P. Kumar, "Age and gender assessment through three-dimensional morphometric analysis of maxillary sinus using magnetic resonance imaging," Journal of Forensic Dental Sciences, vol. 9, no. 1, p. 46, 2017.

[4] Y. Ariji, T. Kuroki, S. Moriguchi, E. Ariji, and S. Kanda, “Age changes in the volume of the human maxillary sinus: a study using computed tomography," Dento maxillo facial radiology, vol. 23, no. 3, pp. 163-168, 1994.

[5] Y. Lu, Z. Liu, L. Zhang et al., "Associations between maxillary sinus mucosal thickening and apical periodontitis using conebeam computed tomography scanning: a retrospective study," Journal of Endodontia, vol. 38, no. 8, pp. 1069-1074, 2012.

[6] L. Ritter, J. Lutz, J. Neugebauer et al., "Prevalence of pathologic findings in the maxillary sinus in cone-beam computerized tomography," Oral Surgery, Oral Medicine, Oral Pathology, Oral Radiology, and Endodontics, vol. 111, no. 5, pp. 634640, 2011

[7] W. C. Scarfe, A. G. Farman, and P. Sukovic, "Clinical applications of cone-beam computed tomography in dental practice," Journal of the Canadian Dental Association, vol. 72, no. 1, pp. 75-80, 2006.

[8] J. Al Abduwani, A. B. ZilinSkienede Carvalho, A. L. Ferreira Costa et al., "Investigation on the relationship of dimensions of the maxillary sinus drainage system with the presence of sinusopathies: a cone beam computed tomography study," Archives of Oral Biology, vol. 94, pp. 78-83, 2018.

[9] J. Al Abduwani, L. ZilinSkiene, S. Colley, and S. Ahmed, "Cone beam CT paranasal sinuses versus standard multidetector and low dose multidetector CT studies," American Journal of Otolaryngology, vol. 37, no. 1, pp. 59-64, 2016.

[10] S. Attalla, H. Ads, T. Oo, M. A. Abdalqader, P. A. P. Ramanathan, and K. N. B. K. Zaman, "Gender and race determination of the maxillary sinus among Malaysian population by computed tomography," International Journal of Medical Toxicology and Legal Medicine, vol. 23, no. 1and2, p. 5, 2020.

[11] P. MPC Gunathilake, Y. P. R. D. Yapa, A. J. Pinidiyaarachchi, J. V. Wijayakulasooriya, and R. Ranaweera, "Computerized 3D modeling for rapid artificial bone substitute manufacturing," International Journal of Computer Applications, vol. 119, no. 17 , pp. 1-5, 2015.
[12] R. M. Hamdy and N. Abdel-Wahed, "Three-dimensional linear and volumetric analysis of maxillary sinus pneumatization," Journal of Advanced Research, vol. 5, no. 3, pp. 387-395, 2014.

[13] A. Sharan and D. Madjar, "Maxillary sinus pneumatization following extractions: a radiographic study," The International Journal of Oral \& Maxillofacial Implants, vol. 23, no. 1, pp. 4856, 2008.

[14] Y. Ariji, E. Ariji, K. Yoshiura, and S. Kanda, "Computed tomographic indices for maxillary sinus size in comparison with the sinus volume," Dento Maxillo Facial Radiology, vol. 25, no. 1, pp. 19-24, 1996.

[15] R. M. Hamdy and N. Abdel-Wahed, "Cone-beam computed tomographic volumetric analysis of the maxillary antra for sinus augmentation," Egyptian Dental Journal, vol. 58, no. 4, pp. 3157-3165, 2012.

[16] P. S. Tiwana, G. M. Kushner, and R. H. Haug, "Maxillary sinus augmentation," Dental Clinics of North America, vol. 50, no. 3, pp. 409-424, 2006.

[17] T. Koppe, M. Nakatsukasa, and A. Yamanaka, "Implication of craniofacial morphology for the pneumatization pattern of the human alveolar process," Acta medica Lituanica, vol. 12, no. 1, pp. 40-46, 2005.

[18] Y. Ariji, N. Obayashi, M. Goto et al., "Roots of the maxillary first and second molars in horizontal relation to alveolar cortical plates and maxillary sinus: computed tomography assessment for infection spread," Clinical Oral Investigations, vol. 10, no. 1, pp. 35-41, 2006.

[19] V. Nimigean, V. R. Nimigean, N. Măru, D. I. Sălăvăstru, D. Bădiță, and M. J. Tuculină, "The maxillary sinus floor in the oral implantology," Romanian Journal of Morphology and Embryology, vol. 49, no. 4, pp. 485-489, 2008.

[20] M. Saccucci, F. Cipriani, S. Carderi et al., "Gender assessment through three-dimensional analysis of maxillary sinuses by means of cone beam computed tomography," European Review for Medical and Pharmacological Sciences, vol. 19, no. 2, pp. 185-193, 2015.

[21] T. Georgiev, K. Dzhabalyan, R. Andreeva, and K. Prodanova, "Linear and volumetric assessment of the maxillary sinus," Scripta Scientifica Medicinae Dentalis, vol. 2, no. 1, pp. 6269, 2016. 